\title{
Potential biomarkers of abnormal osseointegration of implants in type II diabetes mellitus
}

Lingxiao Wang ${ }^{1}$, Zhenhua Gao², Changying Liu' and Jun $\mathrm{Li}^{1}{ }^{1 *}$

\begin{abstract}
Background: Type II diabetes mellitus (T2DM) is an important risk factor for osseointegration of implants. The aim of this study was to explore key genes of T2DM affecting bone metabolism through bioinformatic analysis of published RNA sequencing data, identify potential biomarkers, and provide a reference for finding the molecular mechanism of abnormal osseointegration caused by T2DM.

Methods: We identified differentially expressed mRNAs and miRNAs from the Gene Expression Omnibus database using the R package 'limma' and analysed the predicted target genes using Kyoto Encyclopedia of Genes and Genomes pathway enrichment analysis and Gene Ontology analysis. At the same time, miRNA-mRNA interactions were explored using miRWalk 2.0.
\end{abstract}

Results: We constructed an miRNA-gene regulatory network and a protein-protein interaction network. The enrichment pathways of differentially expressed mRNAs included extracellular matrix receptor interactions, protein digestion and absorption, the PI3K-Akt signalling pathway, cytokine-cytokine receptor interactions, chemokine signalling pathways, and haematopoietic cell lineage functions. We analysed the expression of these differentially expressed mRNAs and miRNAs in T2DM rats and normal rats with bone implants and identified Smpd3, Itga10, and rno-mir-207 as possible key players in osseointegration in T2DM.

Conclusion: Smpd3, Itga10, and rno-mir-207 are possible biomarkers of osseointegration in T2DM. This study sheds light on the possible molecular mechanism of abnormal osseointegration caused by bone metabolism disorder in T2DM.

Keywords: Bioinformatics analysis, Biomarkers, Osseointegration, miRNAs, T2DM

\section{Background}

With the development of dental implant technology and materials, implant-supported prostheses have become the first choice for restoring oral function and aesthetics among patients with missing teeth. Type II diabetes

\footnotetext{
*Correspondence: lijun3021@aliyun.com

1 Department of Dental Implant Center, Beijing Stomatological Hospital, Capital Medical University, Capital Medical University School of Stomatology, No. 4 Tian Tan Xi Li, Beijing 100050, People's Republic of China

Full list of author information is available at the end of the article
}

mellitus (T2DM) is a chronic disease; it has a high prevalence and can have adverse effects on tissues and organs throughout the body [1-3]. T2DM is an important risk factor for periodontal disease and alveolar bone loss. The rate of tooth loss in T2DM patients is 15\% higher than that in healthy people, and the proportion of T2DM patients with dental implants is increasing $[4,5]$. Regarding implant osseointegration, the biological function of alveolar bone-derived cells, the blood supply of the alveolar bone, and the metabolic status of bone tissue all play important roles in implant osseointegration. Previous

(c) The Author(s) 2021. Open Access This article is licensed under a Creative Commons Attribution 4.0 International License, which permits use, sharing, adaptation, distribution and reproduction in any medium or format, as long as you give appropriate credit to the original author(s) and the source, provide a link to the Creative Commons licence, and indicate if changes were made. The images or other third party material in this article are included in the article's Creative Commons licence unless indicated otherwise in a credit line to the material. If material is not included in the article's Creative Commons licence and your intended use is not permitted by statutory regulation or exceeds the permitted use, you will need to obtain permission directly from the copyright holder. To view a copy of this licence, visit http://creativecommons.org/licenses/by/4.0/. The Creative Commons Public Domain Dedication waiver (http://creativeco mmons.org/publicdomain/zero/1.0/) applies to the data made available in this article, unless otherwise stated in a credit line to the data. 
studies have shown that the proliferation and osteogenic differentiation abilities of osteoblasts are weaker in patients with diabetes mellitus than in healthy people. Human blood carries a large amount of active protein and oxygen that can affect the state of bone-derived cells around the implant, and T2DM is often accompanied by high levels of glucose [6,7]. Patients with diabetes often have accompanying calcium and phosphorus metabolism disorders, which affect bone tissue remodelling and interfere with implant-bone bonding, especially $1-3$ weeks after implant placement. Bone tissue remodelling disorders caused by diabetes are important risk factors for the failure of oral implants [8-10].

At present, identifying the molecular mechanisms underlying bone tissue remodelling disorders around dental implants in diabetes patients is an area of active research. Several studies have shown that microRNAs (miRNAs) play an important role in the occurrence and development of bone tissue remodelling around dental implants [11-14]. miRNAs are single-stranded non-coding RNA molecules with approximately 20-24 nucleotides. They are highly evolutionarily conserved and are mainly involved in post-transcriptional regulation. Recent studies have confirmed that miRNAs can inhibit mRNA expression or reduce mRNA stability by specifically binding to the $3^{\prime}$-untranslated regions of target mRNAs, thus, regulating the expression of related genes. miRNAs play an important role in cell proliferation, differentiation, and apoptosis, and some studies have confirmed that several miRNAs are involved in osteogenic differentiation and bone metabolism-related diseases that affect bone tissue remodelling and new bone formation [15-18].

miRNA-mRNA networks have been widely used to study pathways of various cancers and cardiovascular and neurodegenerative diseases [19-27]. However, miRNAmRNA networks have not been assessed when determining how diabetes affects implant osseointegration. The main difficulty stems from the fact that implant osseointegration is a biological process in which bone tissue and the implant's titanium metal surface directly come into contact to produce a stable combination. The effects of the implant are continuously transmitted and dispersed in the bone tissue, in terms of not only bone formation but also physiological bone absorption and bone maturation. At the cellular level, this mainly involves the multidirectional regulation of osteoblasts, osteoclasts, and macrophages, among other cell types. Accurately manipulating the molecular regulatory network while in the process of implant-bone binding is key to constructing a miRNA-mRNA network for this process [28-34]. One study found that when inserting an implant in bone, a controlled partial fracture is created, and the fracture healing process is very similar to that following implant placement. In both osseointegration and fracture healing, substantial bone formation is followed by bone resorption and maturation [35]. Overall, the purpose of this study was to identify miRNAs and mRNAs that could be used as potential predictive biomarkers or therapeutic targets for bone metabolism disorders caused by T2DM. Therefore, in this study, we used callus tissue to identify differentially expressed (DE) mRNAs and miRNAs between rats and those with T2DM. Moreover, we used miRWalk 2.0 to study miRNA-mRNA interactions, established an miRNA-mRNA network, and constructed an implantation model of T2DM rats to verify the DE miRNAs and mRNAs.

\section{Methods}

\section{Collection of RNA-seq datasets}

The Gene Expression Omnibus (GEO, https://www. ncbi.nlm.nih.gov/geo) database was searched to identify all datasets that evaluated mRNA and miRNA expression in bone tissue samples of T2DM animal models. The following terms from the medical subject headings thesaurus were used for the search: ("diabetes" OR "diabetic" OR "T2DM" OR "diabetes mellitus") AND ("miRNA" OR "mRNA") AND ("bone tissue"). Finally, two RNA sequencing (RNA-seq) datasets, GSE76364 and GSE76365, were obtained. The details of GSE76364 and GSE76365 are included in Additional file 1: Table S1. GSE76364 only contained mRNA expression data from the bone callus of T2DM rats (Rattus norvegicus), including $10 \mathrm{~T} 2 \mathrm{DM}$ animals and 10 healthy animals. GSE76365 only contained miRNA expression data from the same tissue of the rats.

\section{Identification of DE mRNAs and miRNAs}

The ENSEMBL IDs of samples were converted by annoE 1.0.3 (https://github.com/ChrisLou-bioinfo/AnnoE NSG2GENE), based on GENCODE 31 (19.06.19) [36]. Differential expression analysis was performed using edge R, an $R$ package for differential expression analysis of biological RNA-seq replicates to identify significant $\mathrm{DE}$ mRNAs and miRNAs in samples from T2DM rats. All q-values were corrected for multiple testing according to false discovery rate (FDR). DE mRNAs with an absolute $\log _{2} \mathrm{FC}>2$ and $\mathrm{FDR}<0.05$, and DE miRNAs with an absolute $\log _{2} \mathrm{FC}>1$ and $\mathrm{FDR}<0.05$ were considered significant and were visualized using volcano plots.

\section{Gene ontology (GO) and kyoto encyclopedia of genes and genomes (KEGG) analysis of DE mRNAs}

Significant DE mRNAs were analysed for GO and KEGG enrichment using Omicshare (https://www.omicshare. com). GO was used to describe gene functions across 
three categories: the biological process (BP), cellular component (CC), and molecular function (MF). Findings from GO and KEGG analyses were searched for results at the significance level set at an adjusted $P$ value of $<0.05$.

\section{miRNA-mRNA targeting relationship prediction}

The miRNA-mRNA interactions were predicted by miRWalk 2.0, which incorporates 12 algorithms for prediction (TargetScan, RNAhybrid, RNA22, PITA, PicTar2, miR-Walk, Microt4, miRNAMap, miRDB, mirBridge, miRanda, and miRMap) [37]. The miRNA-mRNA interactions were visualized using Cytoscape 3.7.1 [38].

\section{KEGG analysis for target mRNAs}

Omicshare (https://www.omicshare.com) was used for KEGG analysis of the target mRNAs. The KEGG analysis results were searched for pathways at the significance level adjusted at $P<0.05$.

\section{Construction of an miRNA-mRNA regulatory network}

Based on the analysis of DE miRNAs, DE mRNAs, and enrichment pathways, Cytoscape 3.7.1 was used to visualize the miRNA-mRNA regulatory network.

\section{Ethical statement}

All experiments on animals were performed in accordance with the ARRIVE guidelines 2.0 and the Ethical Guidelines for the Care and Use of Laboratory Animals of the United States National Institutes of Health. The study protocol, including procedures for animal handling and husbandry, was reviewed and approved by the Animal Experiments and Experimental Animal Welfare Committee of Capital Medical University, Beijing, China (protocol number 2017-104).

\section{Animals and treatment}

Ethical approval was granted by the Animal Care and Use Committee of Capital Medical University (approval number 2017-104). All animal experiments were conducted according to the ethical principles of the Animal Care and Use Committee of Capital Medical University. Twenty 2-week-old male Wistar rats (average weight of $300 \pm 20.0 \mathrm{~g}$ ) were purchased from SPF (Beijing) Biotechnology (Beijing, China). They were randomly divided into an experimental group $(\mathrm{n}=10)$ and a control group $(\mathrm{n}=10)$. The sample size was calculated according to the following formula:

$$
\mathrm{n}=\frac{\left(Z_{\alpha}+Z_{\beta}\right)^{2} * 2 \sigma^{2}}{\delta^{2}} .
$$

The rats were housed under controlled conditions $\left(25{ }^{\circ} \mathrm{C}\right.$ and a 12 -h light/dark cycle) and had ad libitum access to water and rat chow (SPF [Beijing] Biotechnology). The experimental group was fed with diabetesspecific induction feed (SBF [Beijing] Biotechnology) for 7 weeks and were then given a single intraperitoneal injection $(40 \mathrm{mg} / \mathrm{kg})$ of streptozotocin (STZ). Fasting blood glucose was measured on days $0,3,6,9,12,15$, 18, 21, 24, 27, and 30 after STZ treatment. Three days after STZ treatment, animals with fasting blood glucose levels $\geq 16.7 \mathrm{mmol} / \mathrm{L}$ were considered to have diabetes (Additional file 1: Figure S1) [39]. A group of vehicle control rats were injected with an equal volume of $0.1 \mathrm{M}$ citrate buffer. The left leg of each rat was shaved and disinfected. Sterile gauze was laid on the left leg, and the skin was cut approximately $5 \mathrm{~mm}$ from the proximal end of the femoral metaphysis with a diameter of $1 \mathrm{~mm}$ and a depth of $2 \mathrm{~mm}$ by using a minimally invasive approach with water cooling to prepare it for implant placement. After the operation, all rats received a subcutaneous injection of $0.5 \mathrm{mg} / \mathrm{kg}$ ketoprofen.

\section{Euthanasia procedures for experimental animals and sampling technique}

We anaesthetised the animals with $12 \mathrm{mg} / \mathrm{kg}$ of xylazine and $80 \mathrm{mg} / \mathrm{kg}$ of ketamine via intraperitoneal injection and then euthanised them by exsanguination after 7, 14, or 21 days. Under anaesthesia, the abdominal aortas of rats were punctured to collect $8 \mathrm{~mL}$ of blood into heparinised tubes. The leg bone with the implant was collected, the bone tissue around the implant was carefully removed with a wire saw and implant motor (ensuring that $1 \mathrm{~mm}$ of bone tissue remained around the implant); we then carefully scraped off the $1 \mathrm{~mm}$ of bone tissue around the implant with a scraper and put it into TRIzol reagent (Invitrogen, Carlsbad, CA). After that, the animal remains were disposed of in an incinerator.

\section{RT-qPCR analysis}

For analysis of mRNA levels, total RNA from the 1-mm layer of femur tissues around implants were prepared and isolated using TRIzol reagent (Invitrogen). Complementary DNA was synthesised using the RevertAid First Strand Complementary DNA Synthesis kit (Thermo Fisher Scientific, Waltham, MA). Quantitative realtime PCR was performed using the SYBR Premix Ex TaqII (Takara, Dalian, China), as per the manufacturer's instructions. U6 and GAPDH were used as internal controls. Relative expression was calculated using the $2^{-\mathrm{ddCt}}$ method. The primer sequences used are shown in Additional file 1: Table S2.

\section{Receiver operating characteristic (ROC) curves}

ROC curves were constructed to distinguish DE hub genes and miRNAs in the plasma of T2DM rats and 
normal rats, and the areas under the ROC curves (AUCs) were analysed to evaluate the diagnostic accuracy. All statistical analyses were performed using SPSS 13.0 (IBM, Armonk, NY).

\section{Statistical analyses}

A Student's $t$ test was used to compare test and control samples in GraphPad Prism 7 (GraphPad Software, San Diego, CA). Data are presented as means \pm standard deviations for quantitative variables. Mean values of quantitative variables were evaluated by a Student's $t$ test or the Mann-Whitney $U$ test when the requirements for a Student's $t$ test were not met. A $P$ value of $\leq 0.05$ was considered statistically significant. All statistical analyses were performed using SPSS 13.0 (IBM).

\section{Results}

Heterogeneity test and preliminary screening of $D E$ mRNAs and miRNAs

Two RNA-seq datasets (GSE76364 and GSE76365) were included in our study. These comprised 280 significant
DE mRNAs, including 173 upregulated and 107 downregulated mRNAs, in GSE76364 (Fig. 1a), and 13 significant DE miRNAs in GSE76365, including 4 upregulated and 9 downregulated miRNAs (Fig. 1b).

\section{GO and KEGG analyses of significant DE mRNAs}

The significantly upregulated and downregulated DE mRNAs of GSE76364 were utilized for GO and KEGG analyses. On analysing the GO terms of the GSE76364 dataset, the three most enriched BP terms of the downregulated DE mRNAs were "skeletal system development," "ossification," and "extracellular matrix organization," and the three most enriched BP terms of the upregulated DE mRNAs were "immune response," "immune system process," and "defence response." The three most enriched MF terms of the downregulated DE mRNAs were "extracellular matrix structural constituents," "transition metal ion binding," and "fibroblast growth factor-activated receptor activity cytokine activity," and the most enriched MF terms of the upregulated DE mRNAs were "chemokine activity" and "chemokine

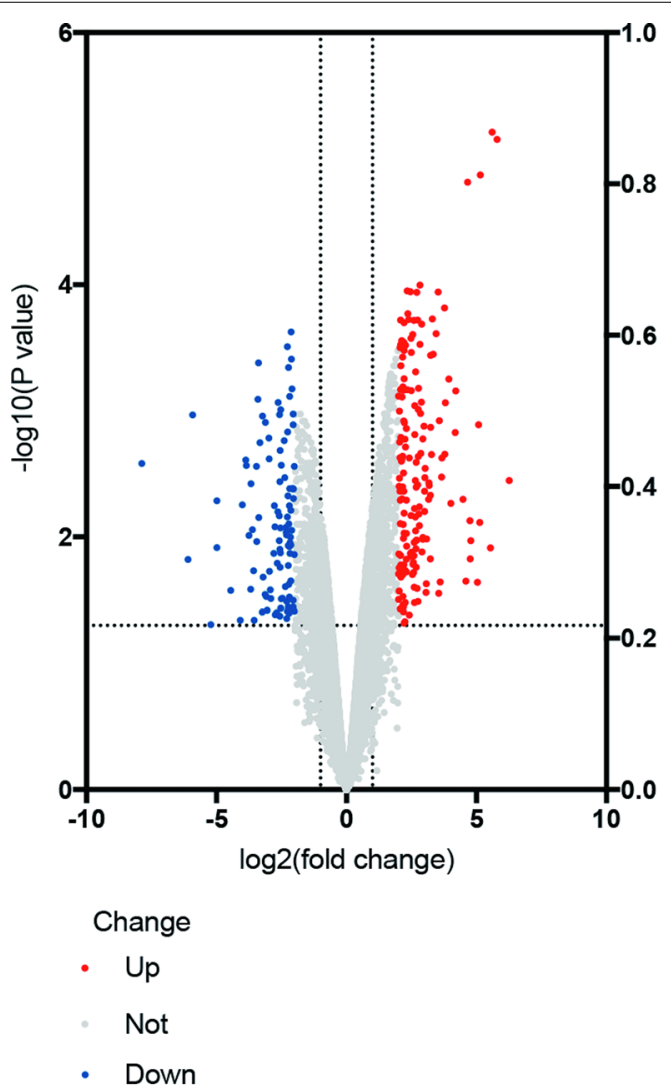

(a)

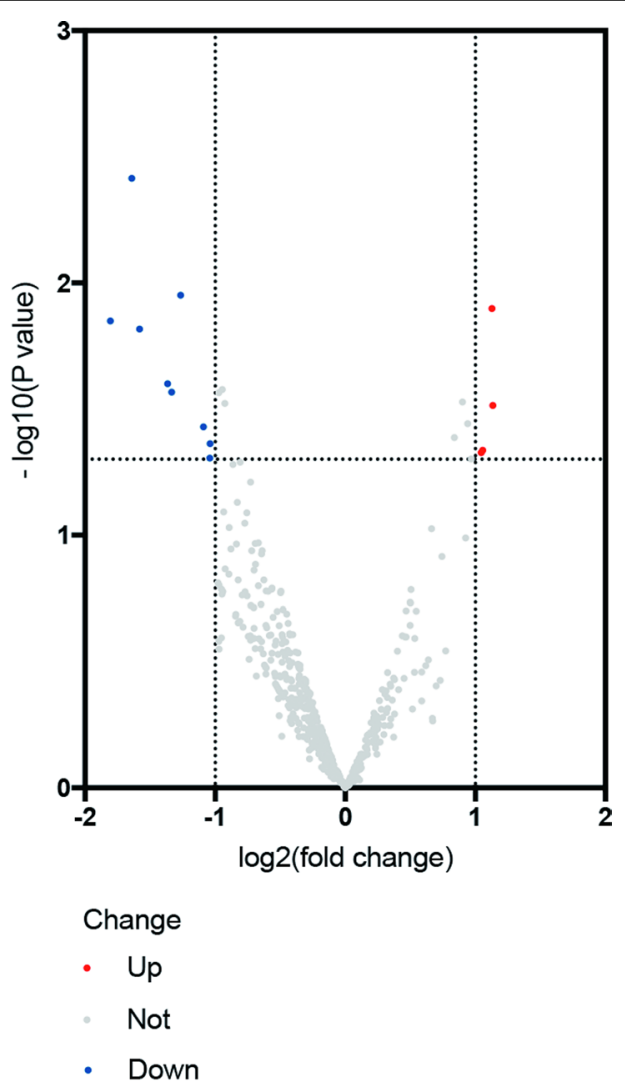

(b)

Fig. 1 The volcano plot of significant differentially expressed mRNAs and miRNAs in GSE76364 and GSE76365 (a) and GSE76364 (b). Red dots represent upregulated mRNAs and miRNAs, and blue dots represent downregulated mRNAs and miRNAs 
receptor binding." The three most enriched CC terms of the downregulated DE mRNAs were "extracellular matrix," "extracellular region," and "extracellular region part," and the three most enriched CC terms of the upregulated DE mRNAs were "extracellular region," "extracellular space," and "extracellular region part" (Fig. 2).

In the KEGG pathway enrichment analysis of the GSE76364 dataset, extracellular matrix (ECM)-receptor interaction, protein digestion and absorption, and the PI3K-Akt signalling pathway were enriched in downregulated DE mRNAs. In the upregulated DE mRNAs, cytokine-cytokine receptor interaction, chemokine signalling pathways, and haematopoietic cell lineage functions were the three most enriched pathways (Fig. 3).

\section{DE miRNA target gene prediction}

We constructed an miRNA-mRNA regulatory network of 13 miRNAs (four upregulated, nine downregulated; Additional file 1: Table S3) and 719 predicted target genes. The number of genes around an miRNA or the number of miRNAs around a surrounding gene was defined as degrees: the more important the network, the greater the degree of its base. Three miRNAs, including $m i R-3557-3 p \quad($ degree $=178), \quad m i R-185-3 p$ $($ degree $=97)$, and $m i R-207($ degree $=80)$, were the core regulatory network miRNAs (Fig. 4).

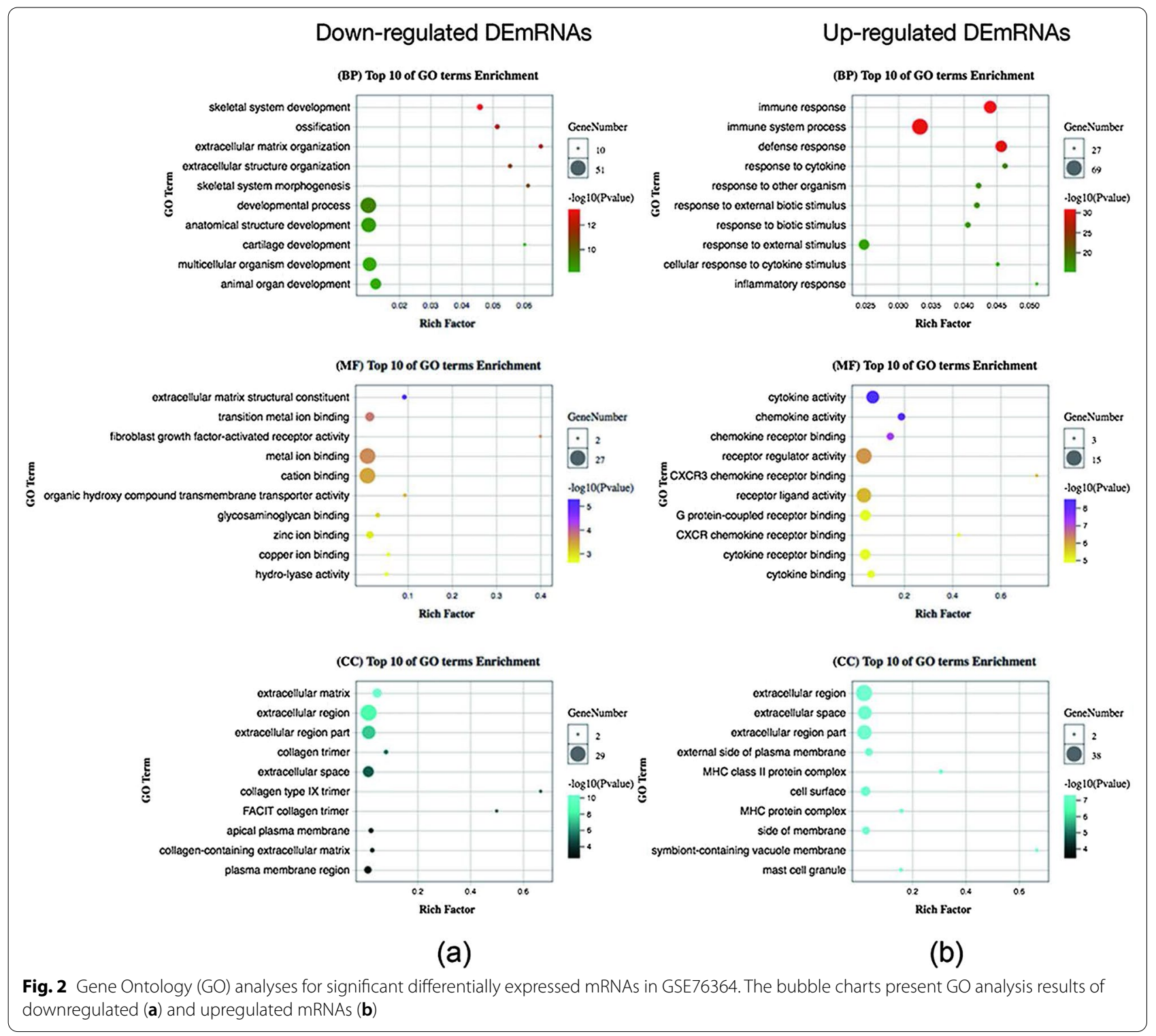


(a) KEGG enrichment barplot

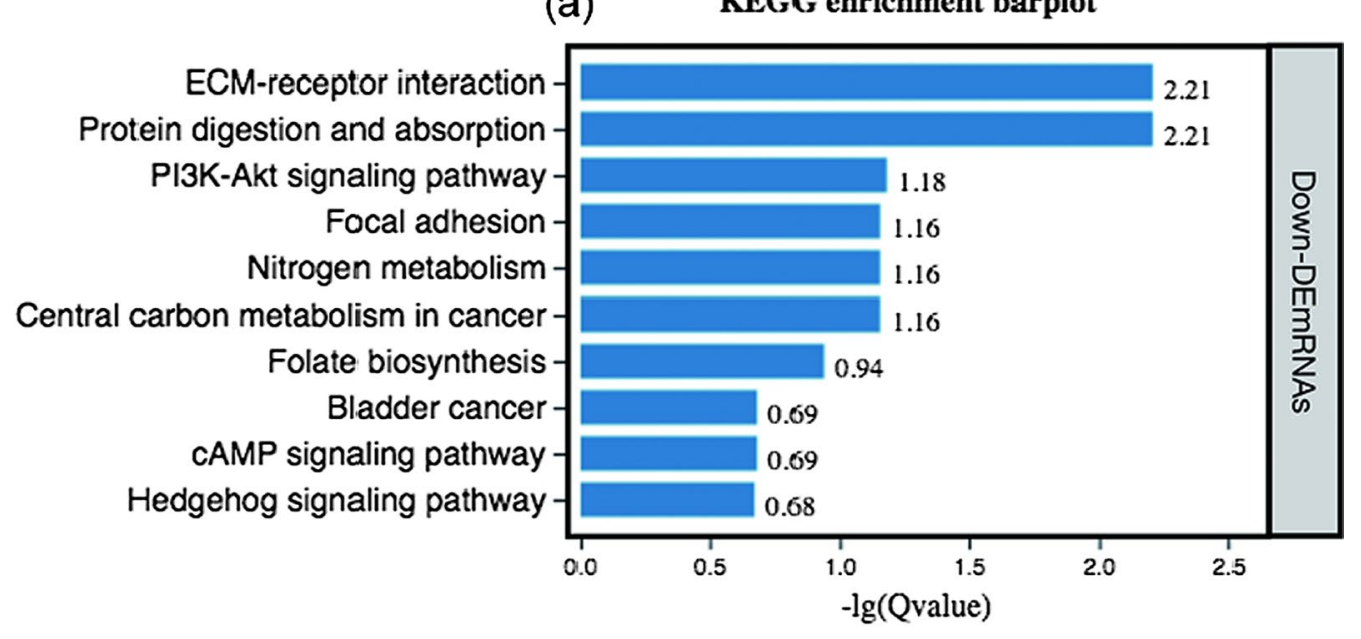

(b) KEGG enrichment barplot

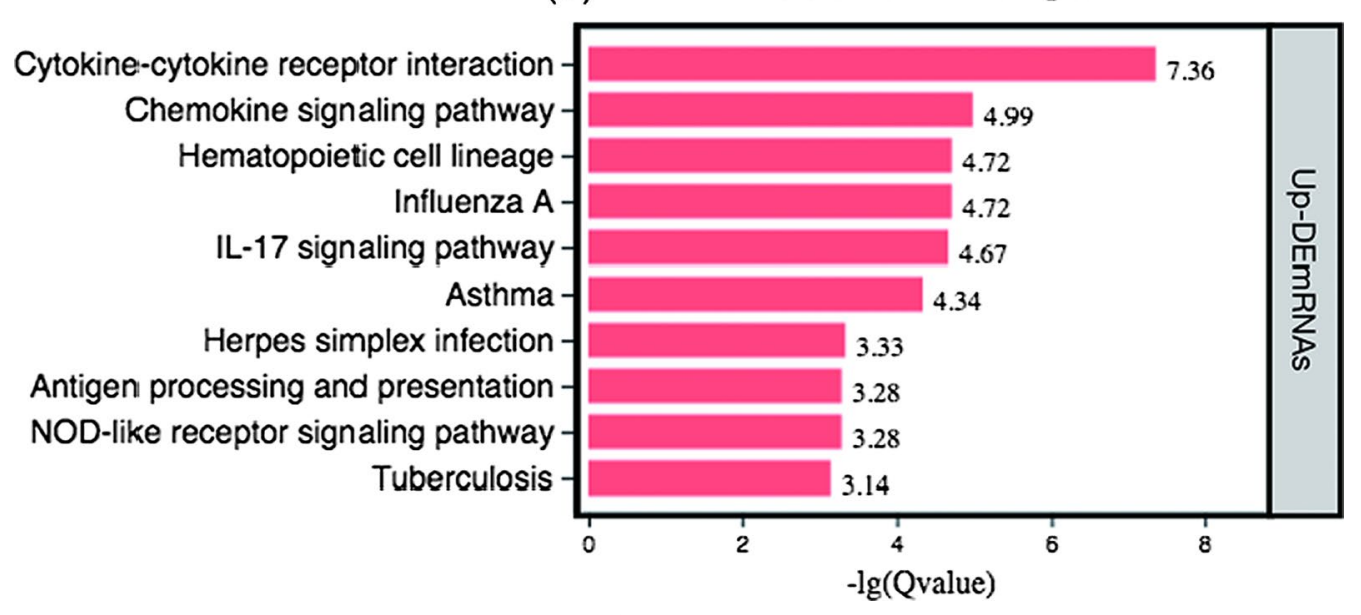

Fig. 3 Kyoto Encyclopedia of Genes and Genomes (KEGG) analyses for significant differentially expressed mRNAs in GSE76364. The bar charts present KEGG analysis results of downregulated (a) and upregulated mRNAs (b)

Intersecting DE miRNA target genes with DE mRNAs and constructing a protein-protein interaction (PPI) network

The six intersecting genes, including Smpd3, Itga10, Slc16a3, Gca, Xkr4, and Mx2 (Additional file 1: Table S4), were obtained by intersecting the $280 \mathrm{DE}$ mRNAs with 719 target mRNAs (Fig. 5a). In the KEGG pathway enrichment analysis of the six genes, dilated cardiomyopathy, hypertrophic cardiomyopathy, and ECM-receptor interaction were the three most enriched pathways (Fig. 5b). Using the STRING database to predict gene interactions, we set a high confidence level (0.700) for the minimum required interaction score and constructed a PPI network (Fig. 5c) of the DE genes in Cytoscape. The average node degree was 12.7, and the top six degrees were Gnb2l1 (degree=19), Stat1 (degree=15), Rps15a
$($ degree $=15), \operatorname{Rps} 23($ degree $=15), \operatorname{Rps} 3($ degree $=15)$, and Rps5 (degree $=15)$.

\section{Validation of the identified miRNAs and mRNA}

During the study period, none of the animals died. miRNA and mRNA levels in the implants were evaluated by quantitative PCR. As shown in Fig. 6, the expression of Smpd3 and Itga10 in the T2DM group decreased 14 and 21 days after implantation, respectively, while the expression of rno-mir-207 increased (Fig. 6a-k). To study the effectiveness of Smpd3, Itga10, and rno-mir-207 as potential biomarkers of T2DM-induced abnormal bone binding, we analysed the ROC curves of these genes in T2DM and normal rats. The AUC values of Smpd3, Itga10, and rno-mir-207 were 0.7037, 0.8395, and 0.9012, respectively (Fig. $7 \mathrm{a}-\mathrm{c}$ ), which were all greater than 0.7 . 


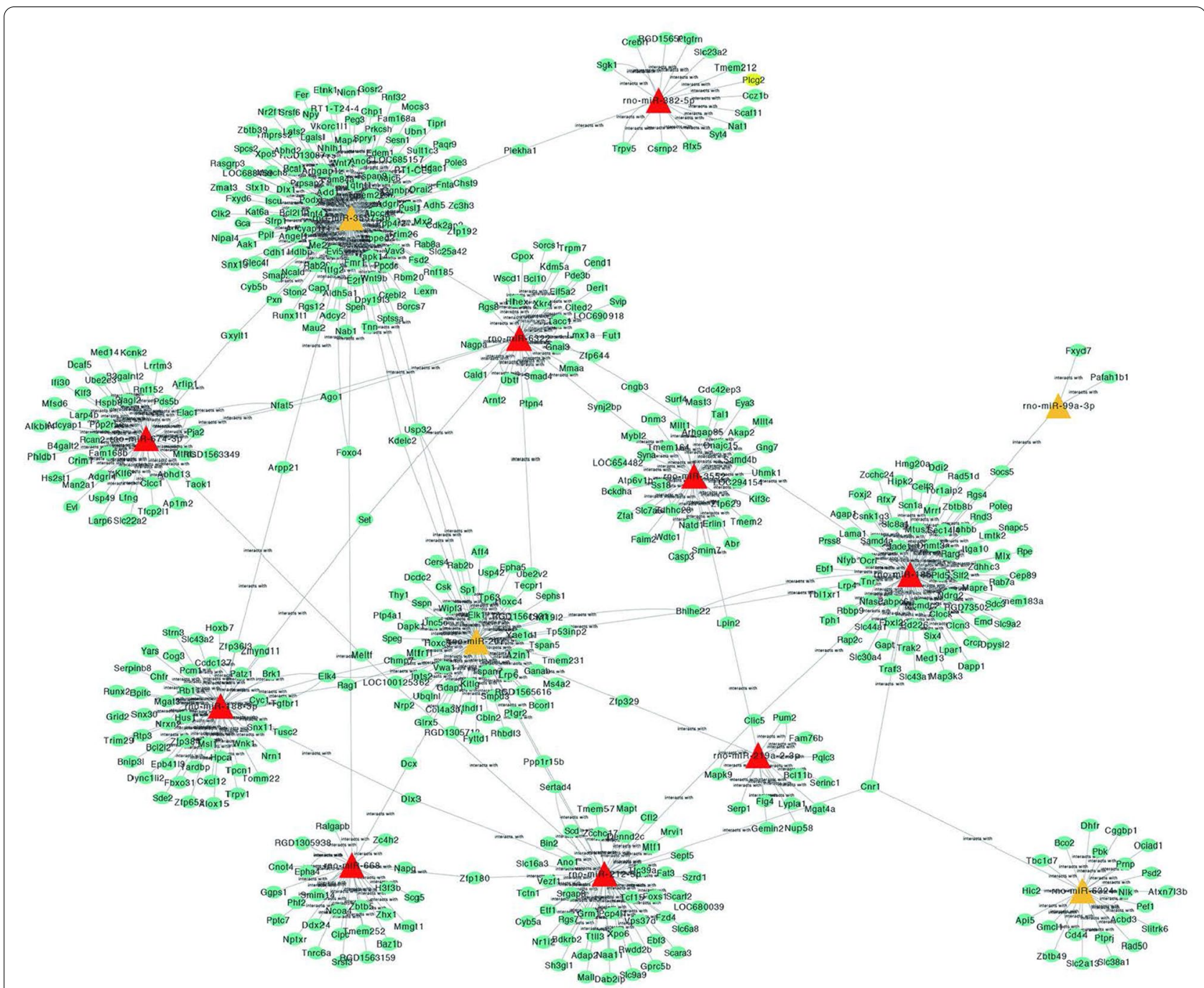

Fig. 4 miRNA-mRNA regulatory network. The relationship between 13 key miRNAs and 719 target genes. Triangle, miRNAs. Ellipse, genes. Yellow, overexpression. Red, downregulation. Straight lines, regulatory relationship between miRNAs and genes

An AUC value higher than 0.7 indicates high accuracy [40]. These results indicate that Smpd3, Itga10, and rnomir-207 have the potential for clinical application.

\section{Discussion}

In 2017, approximately 451 million patients worldwide were reported to have diabetes, and this number is estimated to increase to over 592 million by 2035 . With the increase in the prevalence of diabetes, its various complications will impose a large economic burden on the world [41, 42]. In the field of dental implantology, it has been confirmed that diabetes can cause metabolic disorders of the bone tissue, ultimately leading to failure in implant osseointegration [43-47]. In recent years, with the development of basic research on T2DM and its related metabolic diseases, miRNAs have shown great potential as biomarkers of bone tissue metabolism-related diseases [48-51]. To further study the biological effects of miRNA-mRNA interactions on bone metabolism in patients with diabetes, we constructed an miRNA-mRNA network, including 13 miRNAs and 6 key mRNAs. In addition, we established a rat model of T2DM, verified the hub genes and target miRNAs, and found that Smpd3, Itga10, and rno-mir-207 may be potential biomarkers of abnormal bone binding induced by T2DM.

In our study, we used a multi-step method to identify DE mRNAs in callus tissue from T2DM rats and performed functional and pathway enrichment analysis. The significantly enriched pathways included ECM-receptor 

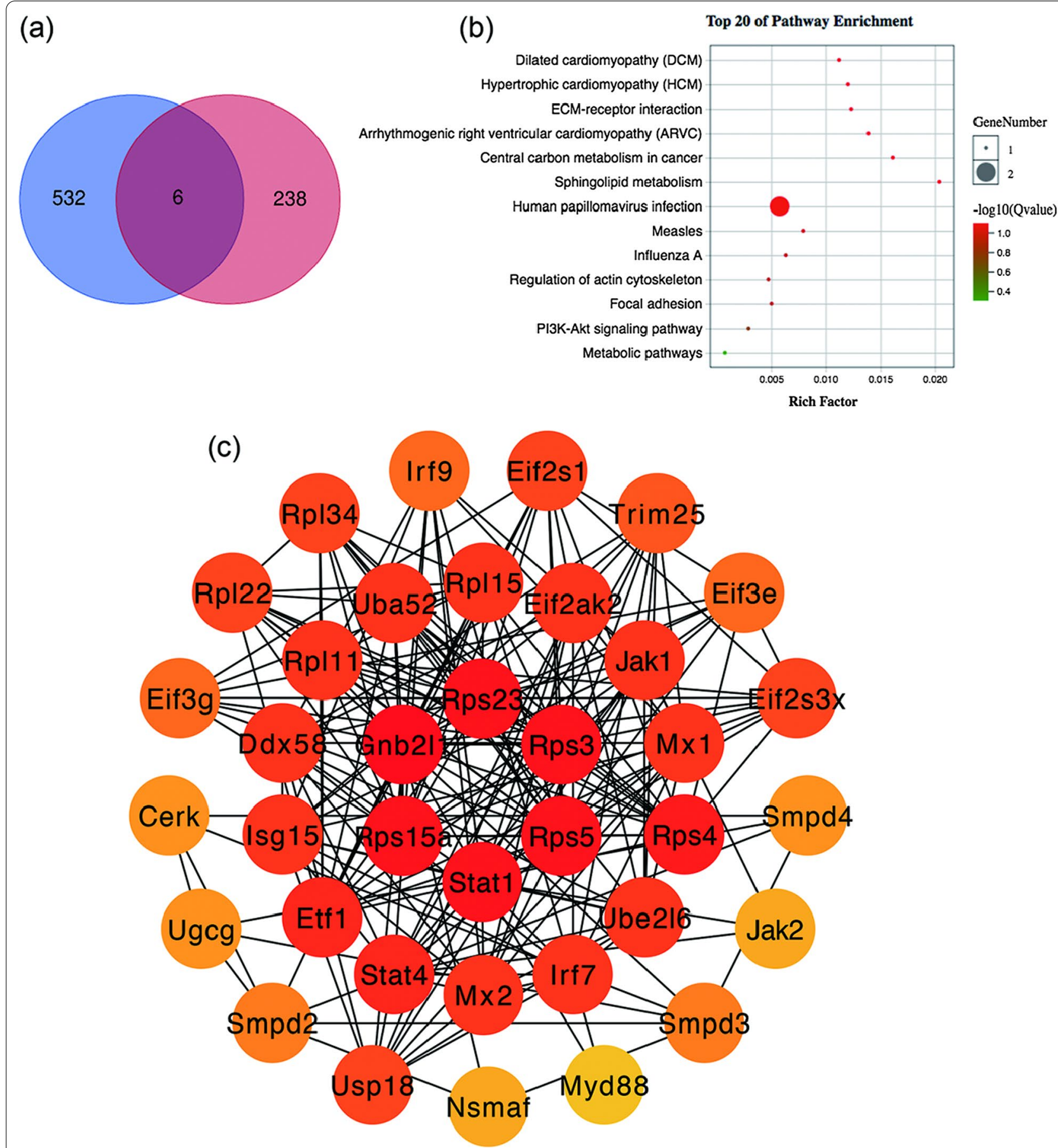

Fig. 5 The six key differentially expressed (DE) mRNAs and protein-protein interaction network. The significant DE mRNAs of GSE104674 and the 13 miRNA-targeted mRNAs are visualized by a Venn plot (a). Kyoto Encyclopedia of Genes and Genomes (KEGG) analyses for six significant DE mRNAs (b). Circles represent genes, and darker colours represent larger degrees. Protein-protein interaction network (c)

interactions, protein digestion and absorption, the PI3K-Akt signalling pathway, cytokine-cytokine receptor interactions, chemokine signalling pathways, and haematopoietic cell lineage functions. The PI3K-Akt signalling pathway plays an important role in bone tissue metabolism, as it can promote the proliferation and differentiation of osteoblasts and participate in the signalling of downstream nuclear factor kappa B receptor 

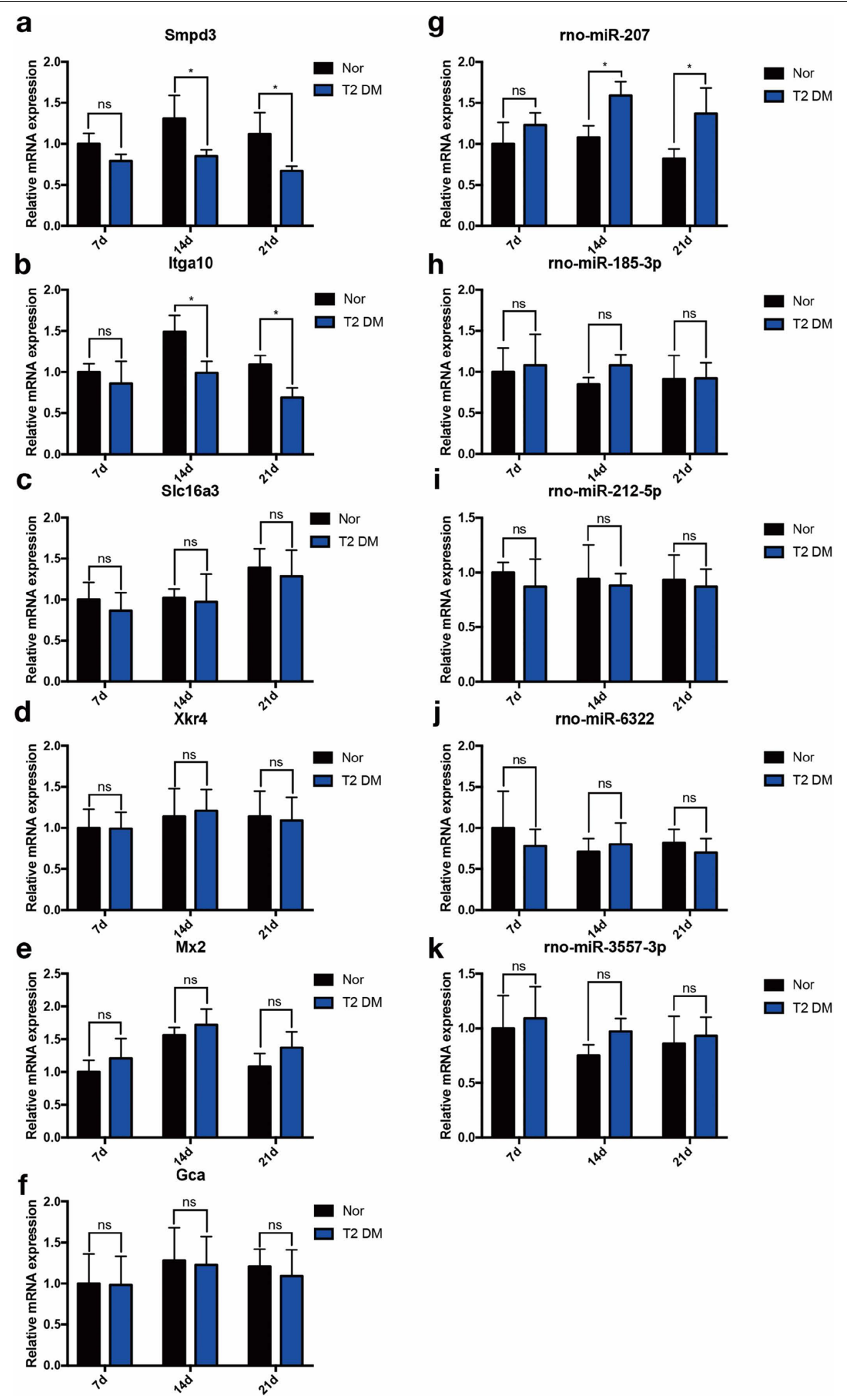

Fig. 6 The relative expression of differentially expressed miRNA and mRNA in bone tissue after implanting 7, 14, and 21 days between T2DM and normal rats. a Smpd3. b Itga10. c Slc16a3. d Xkr4. e Mx2. f Gca. g rno-miR-207. h rno-miR-185-3p. i rno-miR-212-5p. j rno-miR-6322. k rno-miR-3557-3p. ${ }^{*} P<0.05$. T2DM type Il diabetes mellitus 

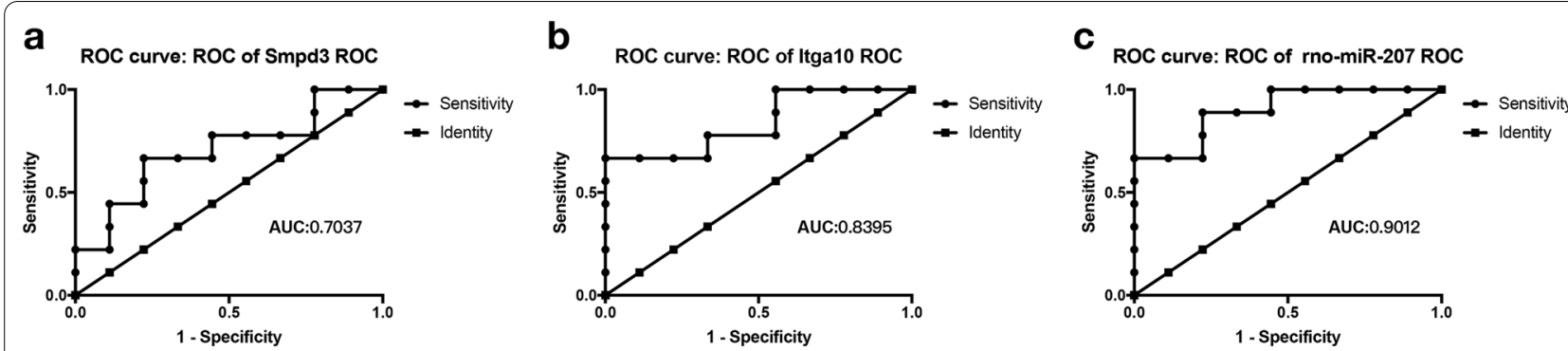

Fig. 7 Receiver operating characteristic curves (ROC) of Smpd3, Itga10, and rno-mir-207 between T2DM and normal rats. a Smpd3, b Itga10, c rno-miR-207. T2DM type II diabetes mellitus

activating factor and macrophage colony stimulating factor receptor. Thus, this pathway plays an important role in osteoclast differentiation and survival, and bone resorption, and inhibiting PI3K-Akt signalling pathway activity will weaken osteoclastic bone resorption $[52,53]$. In addition, the PI3K-Akt-mTOR is a classical pathway in response to insulin signalling. After food consumption, decomposed glucose enters the blood to promote the release of insulin. Insulin, as a signal of nutrient surplus, guides cells to absorb and utilise these nutrients. Insulin first binds to cell-surface receptors and activates the PI3K-Akt pathway through IRS1. Akt directly promotes the absorption of glucose. At the same time, it activates the activity of mTORC1 through Akt-TSC1/2-RhebmTORC1. mTORC1 further guides protein synthesis and uses enzymes related to glucose biosynthesis for nutritional storage [54].

miRNAs can regulate gene expression by inhibiting the translation of target genes or by reducing their stability at the post-transcriptional level. One-third of the human genome can be regulated by miRNAs [55]. Among the selected miRNAs, mir-99a-3p can regulate bone homeostasis and osteoblast differentiation [56]; mir-382-5p is closely related to the decrease of bone mineral density caused by T2DM [57]; miR-185 depletion promotes osteogenic differentiation and suppresses bone loss in osteoporosis through the BGN-mediated BMP/SMAD pathway [58]; and mir-188 can inhibit cell formation, promote fat accumulation in the bone marrow, and it significantly increases in elderly patients [59]. The regulation of cell function is the key to bone formation on the surface of intraosseous implants. As a bridge, non-coding RNAs, especially miRNAs, are directly connected with bone binding as a key factor to control cell function, highlighting the important role of miRNAs in bone binding control. Some emerging in vitro and in vivo studies have emphasised the role of miRNA in bone integration. Many miRNAs affect key bone induction pathways that control OSX, Runx2, and BMP/Smad function, while others affect the monocyte/macrophage lineage. Although great progress has been made in clarifying the mechanism related to the regulation of osteoblast differentiation by miRNAs, those related to bone integration have not been adequately identified and characterised.

In addition, based on RT-qPCR data and ROC analysis, we identified two important hub genes (Smpd3 and Itga10) and an miRNA (rno-mir-207). Smpd3 is expressed in bone and cartilage, and its deficiency leads to cartilage dysplasia and poor bone mineralisation [60-62]. ITGA10 is a transmembrane protein, which is mainly involved in cell adhesion and signal transduction on the cell surface. Studies have shown that ITGA10 can regulate the balance between osteogenesis and osteoclastogenesis and then rescue glucocorticoid-induced osteoporosis [63]. The miRNA rno-mir-207 is downregulated after osteogenic differentiation of bone marrow-derived stromal cells induced by tacrolimus, suggesting that it may play an important regulatory role in osteogenic differentiation of bone marrow-derived stromal cells [64]. Together, these studies suggest that Smpd3, Itga10, and rno-mir-207 may have potential regulatory effects on bone metabolism, but miRNA and mRNA verification is new in the field of oral implant osseointegration and the potential roles of these genes in bone metabolism are unclear. Compared with microarray data, bioinformatics analysis appears to be a promising approach, and it is advantageous as it can create networks using genes, protein-protein interactions, pathway enrichment, and miRNA-mRNA interactions. Here, we show its utility in studying the molecular mechanism of bone tissue metabolism disorder in T2DM.

Our study has some limitations. In the present study, we established a T2DM animal model using the classic STZ injection method. This method is highly repeatable, efficient, and relatively cheap. However, GK rats are a non-insulin-dependent spontaneous T2DM model, which mainly manifests in the islets of Langerhans. These rats feature impaired secretion of $\beta$ cells; increased 
glycogen production; insulin resistance induced by liver, muscle, and adipose tissue; and various non-obesityrelated complications of diabetes, which are all closely related to human T2DM. In addition, the function and molecular mechanisms of genes are complex. Therefore, the genes identified in this study still need to be verified by cell and animal experiments.

\section{Conclusions}

We created an miRNA-mRNA network to further understand the effects of diabetes on bone metabolism. In this network, miRNAs target and regulate the expression of mRNAs, and this affects the occurrence and development of metabolic disorders in the bone tissue of patients with T2DM. Moreover, in this study, we found that Smpd3, Itga10, and rno-mir-207 may be potential biomarkers of T2DM affecting osseointegration. The results of this study can provide a reference for risk assessment and intervention targets when placing oral implants and in developing further clinical applications of this approach.

\begin{abstract}
Abbreviations
T2DM: Type II diabetes mellitus; RT-qPCR: Quantitative real-time PCR; mRNA: Messenger RNA; miRNAs: MicroRNAs; PI3K: Phosphatidylinositol 3-kinase; DE: Differentially expressed; GEO: Gene Expression Omnibus; GO: Gene Ontology; KEGG: Kyoto Encyclopedia of Genes and Genomes; BP: Biological process; CC: Cellular component; MF: Molecular function; STZ: Streptozotocin; ROC: Receiver operating characteristic; AUC: Area under the receiver operating characteristic curve; ECM: Extracellular matrix; PPI: Protein-protein interaction; Smpd3: Sphingomyelin phosphodiesterase 3; Itga 10: Integrin subunit alpha 10; Slc16a3: Solute carrier family 16 member 3; Gca: Grancalcin; Xkr4: XK-related 4; Mx2: MX dynamin like GTPase 2; rno: Rattus norvegicus; BMP: Bone morphogenetic protein.
\end{abstract}

\section{Supplementary Information}

The online version contains supplementary material available at https://doi. org/10.1186/s12903-021-01939-9.

Additional file 1. Additional information.

\section{Acknowledgements}

Not applicable.

\section{Authors' contributions}

All authors contributed to the study conception and design. Material preparation, data collection, and analysis were performed by LXW, ZHG, CYL, and JL. The first draft of the manuscript was written by LXW and all authors commented on previous versions of the manuscript. All authors read and approved the final manuscript.
\end{abstract}

\section{Funding}

This study was funded by the National Nature Science Foundation of China (Grant 81371115) and the Beijing Municipal Administration of Hospitals "Youth Programme, code: QML20171502."

\section{Availability of data and materials}

The data sets used and/or analysed during the current study are available from the corresponding author on reasonable request. Microarray data are deposited in the Gene Expression Omnibus database under the accession code GSE76364 and GSE76365.

\section{Declarations}

Ethics approval and consent to participate

The present study complied with the principles of laboratory animal care and national laws on animal use, and the study was authorized by the Animal Experiments and Experimental Animal Welfare Committee of Capital Medical University, Beijing, China (protocol number 2017-104).

\section{Consent for publication}

Not applicable.

\section{Competing interests}

None of the authors had any competing interests.

\section{Author details}

${ }^{1}$ Department of Dental Implant Center, Beijing Stomatological Hospital, Capital Medical University, Capital Medical University School of Stomatology, No. 4 Tian Tan Xi Li, Beijing 100050, People's Republic of China. ${ }^{2}$ Outpatient Department of Oral and Maxillofacial Surgery, Beijing Stomatological Hospital, Capital Medical University, Capital Medical University School of Stomatology, No. 4 Tian Tan Xi Li, Beijing 100050, People's Republic of China.

Received: 27 January 2021 Accepted: 30 October 2021

Published online: 18 November 2021

\section{References}

1. Petersmann A, Nauck M, Muller-Wieland D, Kerner W, Muller UA, Landgraf R, Freckmann G, Heinemann L. Definition, classification and diagnosis of diabetes mellitus. Exp Clin Endocrinol Diabetes. 2018;126(7):406-10.

2. Guo Y, Huang Z, Sang D, Gao Q, Li Q. The role of nutrition in the prevention and intervention of type 2 diabetes. Front Bioeng Biotechnol. 2020;8:575442.

3. Ren W, Pu F, Luan H, Duan Y, Su H, Fan Y, Jan YK. Effects of local vibration with different intermittent durations on skin blood flow responses in diabetic people. Front Bioeng Biotechnol. 2019;7:310.

4. Dental S, Oberti L, Gabrione F, Nardone M, Di Girolamo M. Two-way relationship between diabetes and periodontal disease: a reality or a paradigm? J Biol Regul Homeost Agents. 2019;33(3 Suppl. 1):153-9.

5. Baeza M, Morales A, Cisterna C, Cavalla F, Jara G, Isamitt $Y$, Pino $P$, Gamonal J. Effect of periodontal treatment in patients with periodontitis and diabetes: systematic review and meta-analysis. J Appl Oral Sci. 2020;28:e20190248.

6. Li J, Liu CY, Jiang YF, Wei XZ, Li JU. Proliferation and differentiation of human osteoblasts from a type 2 diabetic patient in vitro. Genet Mol Res. 2015;14(3):11292-9.

7. Przekora A, Benko A, Blazewicz M, Ginalska G. Hybrid chitosan/beta1,3-glucan matrix of bone scaffold enhances osteoblast adhesion, spreading and proliferation via promotion of serum protein adsorption. Biomed Mater. 2016;11(4):045001.

8. Hua Y, Bi R, Zhang Y, Xu L, Guo J, Li Y. Different bone sites-specific response to diabetes rat models: Bone density, histology and microarchitecture. PLoS ONE. 2018;13(10):e025503.

9. Meza Mauricio J, Miranda TS, Almeida ML, Silva HD, Figueiredo LC, Duarte PM. An umbrella review on the effects of diabetes on implant failure and peri-implant diseases. Braz Oral Res. 2019;33(suppl 1):e070.

10. Ding X, Yang L, Hu Y, Yu J, Tang Y, Luo D, Zheng L. Effect of local application of biphosphonates on improving peri-implant osseointegration in type-2 diabetic osteoporosis. Am J Transl Res. 2019;11(9):5417-37.

11. Menini M, Pesce P, Baldi D, Coronel Vargas G, Pera P, Izzotti A. Prediction of titanium implant success by analysis of microRNA expression in periimplant tissue. A 5-year follow-up study. J Clin Med. 2019;8(6):888.

12. Sartori EM, das Neves AM, Magro-Filho O, Mendonca DBS, Krebsbach PH, Cooper LF, Mendonca G. The role of MicroRNAs in the osseointegration process. Int J Oral Maxillofac Implants. 2019;34(2):397-410.

13. Yan J, Chang B, Hu X, Cao C, Zhao L, Zhang Y. Titanium implant functionalized with antimiR-138 delivered cell sheet for enhanced peri-implant bone formation and vascularization. Mater Sci Eng C Mater Biol Appl. 2018;89:52-64. 
14. Yang C, Liu X, Zhao K, Zhu Y, Hu B, Zhou Y, Wang M, Wu Y, Zhang C, Xu J, et al. miRNA-21 promotes osteogenesis via the PTEN/PI3K/Akt/HIF-1alpha pathway and enhances bone regeneration in critical size defects. Stem Cell Res Ther. 2019;10(1):65.

15. Chen Z, Zhang Y, Liang C, Chen L, Zhang G, Qian A. Mechanosensitive miRNAs and bone formation. Int J Mol Sci. 2017;18(8):1684.

16. Hu R, Li H, Liu W, Yang L, Tan YF, Luo XH. Targeting miRNAs in osteoblast differentiation and bone formation. Expert Opin Ther Targets. 2010;14(10):1109-20.

17. Sera SR, Zur Nieden NI. microRNA regulation of skeletal development. Curr Osteoporos Rep. 2017;15(4):353-66.

18. Zheng J, Zhu L, lok In I, Chen Y, Jia N, Zhu W. Bone marrow-derived mesenchymal stem cells-secreted exosomal microRNA-192-5p delays inflammatory response in rheumatoid arthritis. Int Immunopharmacol. 2020;78:105985

19. Ma L, Shi W, Ma X, Zou M, Chen W, Li W, Zou R, Chen X. Comprehensive analysis of differential immunocyte infiltration and the potential ceRNA networks during epicardial adipose tissue development in congenital heart disease. J TransI Med. 2020;18(1):111.

20. Ma X, Liu C, Gao C, Li J, Zhuang J, Liu L, Li H, Wang X, Zhang X, Dong S, et al. circRNA-associated ceRNA network construction reveals the circRNAs involved in the progression and prognosis of breast cancer. J Cell Physiol. 2020;235(4):3973-83.

21. Qi X, Zhang DH, Wu N, Xiao JH, Wang X, Ma W. ceRNA in cancer: possible functions and clinical implications. J Med Genet. 2015;52(10):710-8.

22. Zhang S, Zhu D, Li H, Li H, Feng C, Zhang W. Characterization of circRNAassociated-ceRNA networks in a senescence-accelerated mouse prone 8 brain. Mol Ther. 2017;25(9):2053-61.

23. Zhang X, Liu Z, Shu Q, Yuan S, Xing Z, Song J. LncRNA SNHG6 functions as a ceRNA to regulate neuronal cell apoptosis by modulating miR-181c-5p/ BIM signalling in ischaemic stroke. J Cell Mol Med. 2019;23(9):6120-30.

24. Zhao Y, Wang H, Wu C, Yan M, Wu H, Wang J, Yang X, Shao Q. Construction and investigation of IncRNA-associated ceRNA regulatory network in papillary thyroid cancer. Oncol Rep. 2018;39(3):1197-206.

25. Huang $R$, Wu J, Zheng Z, Wang G, Song D, Yan P, Yin H, Hu P, Zhu X, Wang $\mathrm{H}$, et al. The construction and analysis of ceRNA network and patterns of immune infiltration in mesothelioma with bone metastasis. Front Bioeng Biotechnol. 2019:7:257.

26. Liu F, Dong H, Mei Z, Huang T. Investigation of miRNA and mRNA co-expression network in ependymoma. Front Bioeng Biotechnol. 2020;8:177.

27. Wang C, Xu X, Chen J, Kang Y, Guo J, Duscher D, Yang X, Guo G, Ren S, Xiong $\mathrm{H}$, et al. The construction and analysis of IncRNA-miRNA-mRNA competing endogenous RNA network of schwann cells in diabetic peripheral neuropathy. Front Bioeng Biotechnol. 2020;8:490.

28. Araujo-Gomes N, Romero-Gavilan F, Garcia-Arnaez I, Martinez-Ramos C, Sanchez-Perez AM, Azkargorta M, Elortza F, de Llano JJM, Gurruchaga M, Goni I, et al. Osseointegration mechanisms: a proteomic approach. J Biol Inorg Chem. 2018;23(3):459-70.

29. Branemark R, Thomsen P. Biomechanical and morphological studies on osseointegration in immunological arthritis in rabbits. Scand J Plast Reconstr Surg Hand Surg. 1997;31(3):185-95.

30. Cooper LF. Biologic determinants of bone formation for osseointegration: clues for future clinical improvements. J Prosthet Dent. 1998;80(4):439-49.

31. Mavrogenis AF, Dimitriou R, Parvizi J, Babis GC. Biology of implant osseointegration. J Musculoskelet Neuronal Interact. 2009;9(2):61-71.

32. Nishimura I. Genetic networks in osseointegration. J Dent Res. 2013;92(12 Suppl):109S-118S

33. D'Aurizio R, Russo F, Chiavacci E, Baumgart M, Groth M, D'Onofrio M, Arisi I, Rainaldi G, Pitto L, Pellegrini M. Discovering miRNA regulatory networks in Holt-Oram syndrome using a zebrafish model. Front Bioeng Biotechnol. 2016:4:60

34. Ran Y, Yin N, Huang D, Zhao Y, Yang J, Zhang H, Qi H. Identification and characterization of circular RNA as a novel regulator and biomarker in preterm birth. Front Bioeng Biotechnol. 2020;8:566984.

35. Eriksson C, Ohlson K, Richter K, Billerdahl N, Johansson M, Nygren H. Callus formation and remodeling at titanium implants. J Biomed Mater Res A. 2007;83(4):1062-9.

36. Harrow J, Frankish A, Gonzalez JM, Tapanari E, Diekhans M, Kokocinski F, Aken BL, Barrell D, Zadissa A, Searle S, et al. GENCODE: the reference human genome annotation for The ENCODE Project. Genome Res. 2012;22(9):1760-74.

37. Sticht C, De La Torre C, Parveen A, Gretz N. miRWalk: an online resource for prediction of microRNA binding sites. PLOS ONE. 2018;13(10):e0206239.

38. ShannonP MA, Ozier O, Baliga NS, Wang JT, Ramage D, Amin N, Schwikowski B, Ideker T. Cytoscape: a software environment for integrated models of biomolecular interaction networks. Genome Res. 2003;11(13):2498-504.

39. Zhang KQ, Tian T, Hu LL, Wang HR, Fu Q. Effect of probucol on autophagy and apoptosis in the penile tissue of streptozotocin-induced diabetic rats. Asian J Androl. 2020;22(4):409-13.

40. Catanzaro MP, Weiner A, Kaminaris A, Li C, Cai F, Zhao F, Kobayashi S, Kobayashi T, Huang Y, Sesaki H, et al. Doxorubicin-induced cardiomyocyte death is mediated by unchecked mitochondrial fission and mitophagy. FASEB J. 2019:33(10):11096-108.

41. Cho NH, Shaw JE, Karuranga S, Huang Y, da Rocha Fernandes JD, Ohlrogge AW, Malanda B. IDF Diabetes Atlas: global estimates of diabetes prevalence for 2017 and projections for 2045. Diabetes Res Clin Pract. 2018:138:271-81.

42. Guariguata L, Whiting DR, Hambleton I, Beagley J, Linnenkamp U, Shaw JE. Global estimates of diabetes prevalence for 2013 and projections for 2035. Diabetes Res Clin Pract. 2014;103(2):137-49.

43. Bandeira E, Neves AP, Costa C, Bandeira F. Association between vascular calcification and osteoporosis in men with type 2 diabetes. J Clin Densitom. 2012;15(1):55-60.

44. Issa C, Zantout MS, Azar ST. Osteoporosis in men with diabetes mellitus. J Osteoporos. 2011;2011:651867.

45. Kurra S, Siris E. Diabetes and bone health: the relationship between diabetes and osteoporosis-associated fractures. Diabetes Metab Res Rev. 2011:27(5):430-5.

46. Valkusz Z. Diabetes and osteoporosis. Orv Hetil. 2011;152(29):1161-6.

47. Wongdee K, Charoenphandhu N. Osteoporosis in diabetes mellitus: possible cellular and molecular mechanisms. World J Diabetes. 2011;2(3):41-8

48. Kim D, Song J, Ahn C, Kang Y, Chun CH, Jin EJ. Peroxisomal dysfunction is associated with up-regulation of apoptotic cell death via miR-223 induction in knee osteoarthritis patients with type 2 diabetes mellitus. Bone. 2014;64:124-31.

49. Qin Y, Ye J, Wang P, Gao L, Wang S, Shen H. miR-223 contributes to the AGE-promoted apoptosis via down-regulating insulin-like growth factor 1 receptor in osteoblasts. Biosci Rep. 2016:36(2):e00314.

50. Takahara S, Lee SY, Iwakura T, Oe K, Fukui T, Okumachi E, Waki T, Arakura M, Sakai Y, Nishida K, et al. Altered expression of microRNA during fracture healing in diabetic rats. Bone Joint Res. 2018;7(2):139-47.

51. Zhang YJ, Ma YS, Xia Q, Yu F, Lv ZW, Jia CY, Jiang XX, Zhang L, Shao YC, Xie WT, et al. MicroRNAmRNA integrated analysis based on a case of welldifferentiated thyroid cancer with both metastasis and metastatic recurrence. Oncol Rep. 2018;40(6):3803-11.

52. Wu SS, Liang QH, Liu Y, Cui RR, Yuan LQ, Liao EY. Omentin-1 stimulates human osteoblast proliferation through PI3K/Akt signal pathway. Int J Endocrinol. 2013;2013:368970.

53. Peng XD, Xu PZ, Chen ML, Hahn-Windgassen A, Skeen J, Jacobs J, Sundararajan D, Chen WS, Crawford SE, Coleman KG, et al. Dwarfism, impaired skin development, skeletal muscle atrophy, delayed bone development, and impeded adipogenesis in mice lacking Akt1 and Akt2. Genes Dev. 2003;17(11):1352-65.

54. Li L, Sun Y, Zhang Y, Wang W, Ye C. Mutant Huntingtin impairs pancreatic beta-cells by recruiting IRS-2 and disturbing the PI3K/AKT/FoxO1 signaling pathway in Huntington's disease. J Mol Neurosci. 2021. https://doi. org/10.1007/s12031-021-01869-9

55. Lewis BP, Burge CB, Bartel DP. Conserved seed pairing, often flanked by adenosines, indicates that thousands of human genes are microRNA targets. Cell. 2005;120(1):15-20.

56. Moura SR, Bras JP, Freitas J, Osorio H, Barbosa MA, Santos SG, Almeida MI. miR-99a in bone homeostasis: regulating osteogenic lineage commitment and osteoclast differentiation. Bone. 2020;134:115303.

57. Heilmeier U, Hackl M, Skalicky S, Weilner S, Schroeder F, Vierlinger K, Patsch JM, Baum T, Oberbauer E, Lobach I, et al. Serum miRNA signatures are indicative of skeletal fractures in postmenopausal women with and without type 2 diabetes and influence osteogenic and adipogenic 
differentiation of adipose tissue-derived mesenchymal stem cells in vitro. J Bone Miner Res. 2016;31 (12):2173-92.

58. Cui $Q$, Xing J, Yu M, Wang Y, Xu J, Gu Y, Nan X, Ma W, Liu H, Zhao H. MmumiR-185 depletion promotes osteogenic differentiation and suppresses bone loss in osteoporosis through the Bgn-mediated BMP/Smad pathway. Cell Death Dis. 2019;10(3):172.

59. Li CJ, Cheng P, Liang MK, Chen YS, Lu Q, Wang JY, Xia ZY, Zhou HD, Cao X, $\mathrm{Xie} \mathrm{H}$, et al. MicroRNA-188 regulates age-related switch between osteoblast and adipocyte differentiation. J Clin Investig. 2015:125(4):1509-22.

60. Liedert A, Rontgen V, Schinke T, Benisch P, Ebert R, Jakob F, Klein-Hitpass L, Lennerz JK, Amling M, Ignatius A. Osteoblast-specific Krm2 overexpression and Lrp5 deficiency have different effects on fracture healing in mice. PLoS ONE. 2014;9(7):e103250.

61. Manickam G, Moffatt P, Murshed M. Role of SMPD3 during bone fracture healing and regulation of its expression. Mol Cell Biol. 2019;39(4):e00370-18
62. Stoffel W, Hammels I, Jenke B, Schmidt-Soltau I, Niehoff A. Neutral sphingomyelinase 2 (SMPD3) deficiency in mice causes chondrodysplasia with unimpaired skeletal mineralization. Am J Pathol. 2019;189(9):1831-45.

63. Huo L, Wang L, Yang Z, Li P, Geng D, Xu Y. Prednisolone induces osteoporosis-like phenotypes via focal adhesion signaling pathway in zebrafish larvae. Biol Open. 2018;7(7):bio029405.

64. Zhang J, Yu X, Yu Y, Gong Y. MicroRNA expression analysis during FK506induced osteogenic differentiation in rat bone marrow stromal cells. Mol Med Rep. 2017;16(1):581-90.

\section{Publisher's Note}

Springer Nature remains neutral with regard to jurisdictional claims in published maps and institutional affiliations.
Ready to submit your research? Choose BMC and benefit from:

- fast, convenient online submission

- thorough peer review by experienced researchers in your field

- rapid publication on acceptance

- support for research data, including large and complex data types

- gold Open Access which fosters wider collaboration and increased citations

- maximum visibility for your research: over 100M website views per year

At BMC, research is always in progress.

Learn more biomedcentral.com/submissions 